\title{
Infección por virus de inmunodeficiencia humana, embarazo y deseo de reproducción. Comité de SIDA Pediátrico Sociedad Chilena de Pediatría
}

\author{
JUANITA ZAMORANO R. y TAMARA HIRSCH B.
}

\author{
Human immunodeficiency infection, pregnancy and family planning. \\ Pediatric AIDS Committee, Chilean Pediatric Society
}

During the last decades there have been great advances in the knowledge of HIV infection with significative impact in the management and prognosis of this disease. Improvement in survival rates and reduction of perinatal transmission are two important facts that have induced changes about recommending pregnancy and family planning to HIV female patients. At present, medical, legal, moral and ethical arguments support HIV positive couples who want to become parents and consider them as carriers of a chronic disease like diabetes mellitus or chronic renal failure.

Key words: AIDS; Pregnancy; Risk; Advise; Vertical transmission.

Palabras claves: SIDA; Embarazo; Riesgo; Consejería; Transmisión vertical.

\section{Introducción}

El SIDA y la infección por el VIH han constituido un fenómeno médico y social en las ultimas décadas del siglo XX y comienzos del XXI; en la actualidad, gran parte de las investigaciones científicas que se desarrollan en el campo médico, están enfocadas a esta patología. Los avances logrados en el conocimiento de esta infección y sus consecuencias han sido notables, determinando cambios significativos en el tratamiento y el pronóstico de las personas infectadas con el VIH. Estos cambios han tenido repercusión en las diferentes situaciones cotidianas de las personas que conviven con el VIH. El embarazo o el deseo de tener hijos entre las mujeres y parejas infectadas con VIH representan una de estas situaciones. En la primera década de conocida la enfermedad por el VIH, la transmisión perinatal fue identificada como la principal causa de SIDA en niños, y las mujeres que se embarazaron o quisieron hacerlo en ese período fueron duramente criticadas, inducidas al aborto o estigmatizadas. En la actualidad, la realidad que enfrentan estas mujeres es muy diferente, con expectativas de vida para ellas mucho mejores por el uso de terapia antiretroviral altamente activa y una reducida posibilidad de transmisión de la infección a su hijo, gracias al tratamiento profiláctico. En este artículo revisaremos los aspectos médicos, éticos y legales relacionados con el embarazo y deseo de reproducción en el grupo de personas que se encuentran infectadas por el VIH y que facilitarán la consejería y toma de decisiones a médicos y a las parejas involucradas

\section{Aspectos médicos}

Epidemiología en Chile: Desde el inicio de la epidemia en 1984 hasta diciembre de 2002 se han

Facultad de Medicina. Universidad de los Andes (JZR).

Departamento de Pediatría Escuela de Medicina Universidad Católica de Chile (THB).

Comité de SIDA Pediátrico de la Sociedad Chilena de Pediatría (JZR, THB)

Recibido: 3 junio 2004

Aceptado: 11 junio 2004 
notificado 5.326 enfermos de SIDA, 5.916 personas infectadas con VIH asintomáticas y 3.494 personas fallecidas. La mayoría son hombres, $89,1 \%$. Durante los últimos años hay un crecimiento relativo de casos de SIDA en mujeres en relación a los casos en hombres, incluyendo todos los mecanismos de transmisión. La razón hombre: mujer muestra una tendencia a la disminución, la brecha entre ambos sexos se acorta indicando un incremento mayor en los casos de VIH-SIDA en mujeres. La diferencia entre el período 90-92 y 96-98 alcanza significación estadística ( $\mathrm{p}=0,00003)$, fenómeno llamado feminización de la epidemia. El 85\% de los casos, se concentra entre los 20 y 49 años. Los menores de 20 años representan $2,3 \%$ y los mayores de 50 , el $12,7 \%$, no existiendo diferencias significativas entre ambos sexos. La forma de transmisión ha cambiado en el tiempo, se ha observado una heterosexualización, es decir, un crecimiento relativo de casos transmitidos por relaciones sexuales entre un hombre y una mujer comparados con los casos transmitidos por relaciones sexuales entre hombres. La tendencia de la distribución de casos en mujeres que declararon exposición heterosexual en comparación a hombres que adquirieron la infección por la vía homo-bisexual, muestra un incremento de las primeras, aumento que tiene una fuerte significación estadística al comparar el período 90-92 versus 96-981.

Estos cambios epidemiológicos son similares a los descritos en otras partes del mundo, donde la epidemia comenzó en homosexuales y drogadictos endovenosos para, posteriormente, hacerse más complejo el panorama comprometiendo a mujeres heterosexuales en edad fértil, como una nueva forma de transmisión ${ }^{2}$.

Avances médicos en terapia antiretroviral. En la búsqueda e investigación de tratamiento del VIH-SIDA se han producido avances importantes en tecnología molecular y desarrollo de fármacos que han permitido entender mejor la patogenia y evolución natural de la infección por VIH y cambiar la evolución de la enfermedad. El primero fue la determinación de la carga viral (ARN-VIH) a mediados de 1990, lo que permitió el monitoreo de las etapas de la infección y establecer pronóstico, a lo que se sumó la mayor disponibilidad de nuevos fármacos antiretrovirales. En 1996 se diseñó una nueva estrategia de tratamiento denominada HAART (Highly active antiretroviral therapy-terapia antiretroviral altamente activa). Con esta terapia se ha logrado una dramática disminución de la mortalidad por SIDA en los últimos 5 años, con un cambio claro de las curvas epidemiológicas ${ }^{2}$. La infección por VIH, de ser una enfermedad letal a corto plazo de diagnosticada, pasó a ser una enfermedad crónica.

Prevención de la transmisión perinatal. Tratamiento de la mujer embarazada. En 1994 un grupo de ensayos clínicos de SIDA en niños (Pediatric Antiretroviral Clinical Trial GroupPACTG) desarrolló el protocolo $076^{3}$ cuyo resultado mostró una disminución estadísticamente significativa de la transmisión vertical, desde 26 a $8,3 \%$ en el grupo de niños y madres tratadas versus un grupo que recibió placebo: En este protocolo se administro zidovudina (AZT) a la madre después de la semana 14 de gestación, durante el parto y a los recién nacidos durante el primer mes de vida. Informes del CDC (Atlanta, E.U.A.) posteriores al uso masivo de este protocolo revelan $80 \%$ de disminución de la transmisión perinatal entre los años 1992 y 1997. Otros fármacos y esquemas han demostrado ser también efectivos ${ }^{4,5}$.

En Chile, la transmisión vertical era de 35,4\% antes de aplicar el protocolo 076, con la implementación de éste la transmisión vertical cayó a $2,4 \%$, correspondiendo la mayoría de los casos observados a tratamientos incompletos (Comité SIDA Pediátrico, datos no publicados). En la experiencia internacional se reportan incluso frecuencias de transmisión menores, aun en etapas avanzadas de la enfermedad ${ }^{6}$.

Carga viral y riesgo de transmisión vertical. Se ha comprobado que mujeres embarazadas con cargas virales indetectables en plasma tienen riesgo de transmisión al niño extremadamente bajo, tanto si están con tratamiento antiretroviral como si no lo reciben ${ }^{7}$; sin embargo, el riesgo siempre existe y se han encontrado otros factores asociados como presencia de otras enfermedades de transmisión sexual, drogadicción, tabaquismo, parto de pretérmino, monitorización fetal invasora, desarrollo de corioamnionitis, y mayor tiempo de rotura de membranas.

Tipo de parto. Reducir la exposición del bebé a las secreciones y sangre maternas durante el período del parto puede prevenir la transmisión perinatal del VIH. La operación cesárea realizada antes de iniciarse el trabajo de parto y de la rotura de membranas disminuye el riesgo de transmisión del VIH en 50\%, comparado con la resolución del parto por vía vaginal, en ciertas poblaciones de mujeres ${ }^{8}$. Un estudio randomizado efectuado en Europa demostró el beneficio de la 
operación cesárea electiva antes de iniciarse el trabajo de parto en mujeres infectadas con VIH, tanto si fueran tratadas con terapia antiretroviral como si no lo recibieran ${ }^{9}$. A pesar que comparativamente al parto vaginal la operación cesárea se asocia a mayor morbilidad, tanto en las mujeres infectadas como en las no infectadas con VIH, en 1999 y 2000 el Colegio Americano de Obstetricia y Ginecología (ACOG) recomendó ofrecer a las mujeres la operación cesárea a las 38 semanas de gestación para reducir el riesgo de transmisión vertical de la infección por $\mathrm{VIH}^{10}$. Estudios recientes han demostrado, frecuencias de transmisión cercanas al $2 \%$ en mujeres con tratamiento antiretroviral y con parto por operación cesárea, sin roturas de membranas, y antes de iniciarse el trabajo de parto ${ }^{11}$.

Fertilidad y VIH. La infección por VIH por sí sola altera la fertilidad de las mujeres sintomáticas y asintomáticas, a lo que se puede sumar otras causas de disminución de la fertilidad, como abuso de sustancias, alcohol, y co-infección con otras enfermedades de transmisión sexual.

Hombres infectados por VIH presentan, con alguna frecuencia, hipogonadismo. Los estudios han demostrado que estos hombres tienen un aumento de los niveles de globulina ligante a hormonas sexuales, independiente del recuento de CD4 y una disminución en los niveles séricos de testosterona, que se acentúa con la inmunosupresión ${ }^{12}$.

Efecto del embarazo en la expresión de la infección por VIH. Durante la gestación en mujeres infectadas y no infectadas se produce una disminución de sus recuentos absolutos de CD4, debido a hemodilución; sin embargo, el porcentaje de CD4 permanece estable. La carga viral permanece estable durante los meses de embarazo en mujeres no tratadas. Los estudios sugieren que el embarazo no tiene efecto adverso en la sobrevida o expresión de la enfermedad en la mujer con infección por VIH. Un reciente meta-análisis confirma estos hechos ${ }^{13}$.

Efecto del VIH en el curso del embarazo. Cualquier enfermedad de base en la madre puede afectar el curso del embarazo y la infección por VIH se ha relacionado con mayor riesgo de aborto, parto prematuro y bajo peso de nacimiento. Pueden contribuir otros hechos que con frecuencia presentan las mujeres infectadas, como son drogadicción, consumo de tabaco y desnutrición, siendo difíciles de separar y cuantificar el peso de cada uno ${ }^{14}$.
Fármacos antiretrovirales y embarazo: ¿riesgo o beneficio?. La zidovudina es el fármaco del cual mejor se conocen los efectos sobre el feto y el niño, por la experiencia adquirida con el protocolo 076. Los efectos adversos fueron mínimos; lo más frecuente fue anemia en el recién nacido que se resolvió espontáneamente en la mayoría de las veces. A cuatro años de seguimiento, no hay evidencias de alteraciones neurológicas o del desarrollo de los niños que la recibieron ${ }^{15}$.

Otros antiretrovirales son conocidos como riesgosos; efavirenz demostró en primates producir malformaciones graves como anencefalia, anoftalmia y fisura palatina. La hidroxi-urea se conoce como teratógeno en especies animales afectando a múltiples órganos. Indinavir produce hiperbilirrubinemia y cálculos renales en neonatos, especialmente si son prematuros.

Por ultimo hay algunos reportes de disfunción mitocondrial en niños no infectados que recibieron análogos de nucleótidos, dos desarrollaron enfermedad neurológica severa y fallecieron.

\section{Aspectos éticos}

Como ya se mencionó, al comienzo de la epidemia por el VIH los médicos recomendaron como medida de control de la infección perinatal, limitar la reproducción de las mujeres infectadas, ya que el riesgo de transmisión vertical era de alrededor de $30 \%$, sumado a la alta probabilidad que el niño nacido de este embarazo quedara huérfano a corto plazo. Esta recomendación no era siempre aceptada por las mujeres cuyo deseo de ser madres no se modifica ante el hecho de saberse infectada por el VIH. En 1996 un grupo multidisciplinario de expertos en infección por VIH y en reproducción, basados en los resultados del protocolo 076 y las nuevas terapias antiretrovirales, recomendó la política de dar consejo "contextualizado" a las parejas con infección por VIH y que éstas tomaran una razonable y reflexiva decisión acerca del embarazo ${ }^{16}$.

Por otro lado, los equipos de estudio de fertilidad fueron enfrentados a parejas discordantes (un cónyuge infectado y el otro no) que solicitaban ayuda. En 1994, el Comité de Ética de la Sociedad Americana de Fertilidad consideró que la infección por VIH era una contraindicación para el tratamiento. En 1997, el Comité de aspectos éticos de la Federación Internacional de Ginecología y Obstetricia (FIGO) planteó que sólo los individuos seronegativos para infección por VIH podían participar en tratamientos de fertilización asistida. 
Los especialistas en fertilidad se han visto enfrentados a tres problemas principales:

Participar en la creación de un niño con infección por VIH. En la actualidad en que la transmisión del VIH a niños cuyas madres reciben tratamiento antiretroviral se ha reducido a $2 \%$ y la resolución del parto se efectúa por operación cesárea, esto no sería un hecho importante ni diferente de otras enfermedades en que sí se asiste a las parejas, por ejemplo, madres con diabetes mellitus insulina dependiente cuyo riesgo de dar a luz un niño con malformaciones congénitas es $10 \%$, o aquellas portadoras de cardiopatía congénita $25 \%$.

Transmisión a la pareja discordante. Existe un riesgo de transmisión del VIH a la madre durante el proceso de inseminación en el caso de ser el hombre el infectado. En hombres infectados por VIH, el virus está presente en el semen como virus libre y también asociado a células no espermáticas. No hay evidencias que el VIH sea capaz de adjuntarse o infectar los espermatozoides. Con la técnica de "lavado de espermios" en que se libera a éstos de líquido seminal y células no espermáticas antes de la inseminación, se reduce la posibilidad de transmisión del virus. Un estudio de 1.000 inseminaciones en 350 parejas discordantes para infección por VIH resultó en 200 embarazos y ningún caso de mujer o niño infectado $^{17}$.

Riesgo de transmisión al personal de salud o a los equipos de laboratorio. El riesgo no debiera ser diferente al de la manipulación de otras muestras y debiera ser mínimo si se cumplen las pautas de Precaución Universal.

\section{Aspectos morales}

La bioética se basa en tres "principios" para analizar estos problemas o dilemas ${ }^{16}$.

Respeto por la autonomía o respeto por las personas: respeto por los valores, preferencias y decisiones de otro ser humano aunque sean diferentes a las propias. Atender una pareja infectada por VIH que contempla el embarazo, significa respeto por su decisión informada para intentar la concepción. Sin embargo, si el respeto por la elección de procrear de una pareja se traduce necesariamente en la obligación de entregar los servicios para reproducción asistida, es aún un tema en debate. El principio de autonomía no es absoluto, particularmente si el ejercicio de la autonomía causa daño o limita la libertad de otros. La pregunta que se nos plantea es ihago bien o mal en relación al producto?
Beneficencia: Como cuidadores de la salud significa: promover el bienestar de otros, y definiéndolo negativamente: la obligación de "no hacer mal" a otros. En relación a reproducción asistida, se plantea nuestra obligación con el niño, ¿estamos haciendo bien o mal asistiendo a la concepción de un niño que pudiera nacer con una enfermedad letal o quedar huérfano prematuramente? Con los tratamientos actualmente disponibles para la mujer infectada por VIH este riesgo es bajo y no diferente al riesgo de presentar malformaciones congénitas que enfrentan los hijos de mujeres no infectadas por VIH.

Adicionalmente y específicamente en fertilización asistida, surge la duda de la beneficencia a la comunidad por el riesgo teórico de contaminar el laboratorio; sin embargo, no hay datos que sustenten este hecho, trabajando en áreas donde se aplican las medidas universales de precaución.

Justicia: Requiere de profesionales médicos, políticas de salud y beneficios no discriminatorios. La pregunta aquí es: ¿estamos restringiendo una terapia a personas sólo por el hecho de estar infectadas por el VIH?, o... ¿por el prejuicio, el estereotipo que tenemos en relación a las personas infectadas por VIH (drogadicta, promiscua, homo/bisexual, color etc.)? Si así fuese estaríamos violando el derecho a la no discriminación de los portadores de VIH.

\section{Aspectos legales}

Este problema varía según las leyes vigentes en cada país. En E.U.A. los pacientes portadores de VIH se acogen a una ley de discapacidad vigente desde 1990; su objetivo es proteger de la discriminación a los individuos con cualquier minusvalía. La discapacidad se define como una disminución de las capacidades físicas o mentales que limiten el desarrollo de las actividades cotidianas normales. La infertilidad y la infección por VIH se acogen a esta ley y ya se han realizado numerosos juicios que han resuelto a favor de los pacientes infectados por $\mathrm{VIH}^{16}$.

En el país no contamos con una ley de discapacidad como la de E.U.A. pero el Articulo Primero de la Constitución de Chile dice que: "los hombres nacen libres e iguales en dignidad $y$ derechos" y menciona a la familia como núcleo fundamental de la sociedad, existen leyes de igualdad ante la ley (Art. $19 \mathrm{~N}^{\circ}$ 2), de derecho a la vida privada y familiar (Art. $19 \mathrm{~N}^{\circ} 4$ ) y derecho a la no discriminación arbitraria (Pactos Internacionales) que protegerían en forma teórica en 
Chile a las personas infectadas por VIH, también respecto de la fertilidad

\section{Conclusiones}

La decisión de tener hijos es tradicionalmente un hecho privado y personal en la relación de pareja. Con el inicio de tratamientos de infertilidad, el equipo médico se integró en la toma de estas decisiones. La infección por VIH constituye un ejemplo de los grandes cambios que experimenta la acción médica en sus aspectos preventivos, diagnósticos, terapéuticos y morales. Al comienzo de la década de los 90', se restringió la reproducción asistida a las parejas afectadas por el VIH. Las consideraciones médicas, de tratamiento, éticas y legales analizadas anteriormente sugieren que ya no es recomendable la exclusión de este grupo de personas de las clínicas de fertilidad y lo adecuado sería dar un consejo "contextualizado" en relación a las etapas de la reproducción, los riesgos conocidos, la necesidad de terapia, el pronóstico vital de los padres y respetar la elección de las parejas portadoras de VIH.

\section{Resumen}

El avance del conocimiento sobre la infección por el virus de la inmunodeficiencia humana durante las últimas décadas ha determinado cambios notables en el tratamiento y pronóstico de la enfermedad. La mejoría de la sobrevida y la disminución de la transmisión perinatal constituyen dos hechos importantes que modifican las recomendaciones sobre el embarazo y deseo de reproducción en mujeres infectadas con este virus. Los argumentos médicos, legales, morales y éticos apoyan a las parejas que conviven con el VIH en su deseo de ser padres y los pone en situación similar a parejas que padecen de otras patologías crónicas como diabetes mellitus o insuficiencia renal.

\section{Bibliografía}

1.- Memoria Anual de CONASIDA. Documento. CONASIDA. Diciembre 2002 Rev Chil Infect 2004; 21: $126-50$

2.- Advancing HIV Prevention: New strategies for a changing epidemic. United States. MMWR Morb Mortal Wkly Rep 2003; 52 (15): 329.

3.- Connor E M, Sperling R S, Gelber R, et al. Reduction of maternal-infant transmission of human immunodeficiency virus type 1 with zidovudine treatment. $\mathrm{N}$ Engl J Med 1994; 331: 1173-80.
4.- Sperling R, Shapiro D, Coombs R, Toods J, Herman S, MC-Sherry G. Maternal viral load zidovudine treatment and the risk of transmission of human immunodeficiency virus type 1 from mothers to infants. N Engl Med J 1996; 335: 1621-9

5.- CDC Task Force recommendations for use of antiretroviral drugs in pregnant women infected with HIV-1 for maternal health and for reducing perinatal HIV-1 transmission in the United States. MMWR Morb Mortal Wkly Rep 1998; 47 (RR-2):1-30

6.- CDC Task Force recommendations for use of antiretroviral drugs in pregnant HIV-1--infected women for maternal health and interventions to reduce perinatal HIV-1 transmission in the United States. MMWR Morb Mortal Wkly Rep 2002; 51 ( ${ }^{\circ}$ RR-18).

7.- Chuachoowong R, Shaffer N, Siriwasin W, Chailsilwattana P, Young N, Mock P, et al. Shortcourse antenatal zidovudine reduces both cervicovaginal human immunodeficiency virus tipe-1 levels and risk of perinatal transmission J Infect Dis 2000; 181 99-106.

8.- The International Perinatal HIV Group. The mode of delivery and the risk of vertical transmission of human immunodeficiency virus type 1. N Engl J Med 1999; 340: $977-87$

9.- The European Mode of Delivery Collaboration. Elective cesarean section versus vaginal delivery in prevention of vertical HIV-1 transmission: a randomised clinical trial. Lancet 1999; 353: 1035-9.

10.- Mofenson L, Lambert J, Stiehm E, Bethel J, Meyer W, Whitehouse $\mathrm{J}$ et al. Risk factor for perinatal transmission of human immunodeficiency virus type 1 in women treated with zidovudine. N Engl J Med 1999; 341: 385-93.

11.- Garcia P, Kalish L, Pitt J, Minkoff H, Quinn T, Burchett $S$, et al. Maternal levels of plasma immunodeficiency virus type 1 an the risk of perinatal transmission N Engl J Med 1999; 341: 394-402.

12..-Laudat A, Blum L, Guechot J, Picard O, Cabene J, Imbert $\mathrm{JC}$ et al Charges in systemic gonadal and adrenal steroids in asymptomatic human immunodeficiency virus-infected men relationship with the CD4 cells count. Eur J Endocrinol 1995; 133: 418-24.

13.- French R, Brocklehurst P. The effect of pregnancy on survival in women infected with HIV: a systematic review of the literature and meta-analysis. $\mathrm{Br} \mathrm{J}$ Obstet Gynecol 1998; 105: 827-35.

14.- Brocklehurst P. Prench R The association between maternal HIV infection and perinatal outcome: a systematic review of the literature and meta-analysis. Br J Obstet Gynecol 1998; 105: 836-48.

15.- Hanson I C, Antonelli T A, Sperlling R S, Oleske J M, Cooper E, Culnane M, et al . Lack of tumors in infants with perinatal HIV-1 exposure and fetal/neonatal exposure to zidovudine. J Acquir Immune Defic Syndr 1999; 20: 463-7.

16.- Drapkin A, Anderson J. Human immunodeficiency virus and assisted reproduction: reconsidering evidence, reframing ethics. Fertil Steril 2001; 75: 343-58.

17.- Chrystie I L, Mullem J E, Braude P R, Rowell P, Williams Eelkington N, et al. Assisted conception in HIV discordant couples: evaluation of semen processing techniques in reducing HIV viral load. J Reprod Immunol 1998; 41: 301-6.

Correspondencia a:

Juanita Zamorano Riquelme

E-mail: jzamorano@uandes.cl 